\title{
Recent Results from the SAMPLE Experiment
}

\author{
Takeyasu M. Ito \\ for the SAMPLE Collaboration
}

W.K.Kellogg Laboratory, California Institute of Technology, Pasadena, CA 91125

\begin{abstract}
The previous two SAMPLE experiments yielded a measurement of the axial $e-N$ form factor $G_{A}^{e}$ substantially different from the theoretical estimate. In order to confirm this observation, a third SAMPLE experiment was carried out at a lower beam energy of $125 \mathrm{MeV}\left(Q^{2}=\right.$ $\left.0.038(\mathrm{GeV} / c)^{2}\right)$ on a deuterium target. The data analysis is now at the final stage and the results are consistent with the theoretical prediction of the axial form factor $G_{A}^{e}$. Also, reevaluation of the background dilution factor and the electromagnetic radiative correction for the $200 \mathrm{MeV}$ deuterium data lead to updated results, which are also consistent with the theoretical prediction.
\end{abstract}

\section{INTRODUCTION}

It is well established that parity-violating (PV) electron scattering can provide a measurement of the neutral weak form factors of the nucleon $[1,2]$. When combined with the information on the known electromagnetic form factors, the information on the neutral weak form factors would allow a separation of the nucleon's form factors into the three contributing flavors of quarks: up, down, and strange [3]. Measuring the contribution from strange quark-antiquark pairs is of special interest because it relates directly to the proton's virtual sea that apparently determines the bulk of its mass. This provided the basis for the SAMPLE project (and other experiments that followed). The primary goal of SAMPLE is to determine the proton's strange magnetic form factor $G_{M}^{s}$ through PV electron scattering at backward angles.

PV electron scattering from the proton at backward angles is not only sensitive to $G_{M}^{s}$, but is also sensitive to the proton's neutral weak axial form factor. The neutral weak axial form factor as measured in electron scattering $G_{A}^{e}$ can potentially receive large electroweak corrections, including the anapole moment, that are absent in neutrino scattering. Determining $G_{A}^{e}$ is not only important for a reliable extraction of $G_{M}^{s}$, but also is interesting on its own. Parity violating quasielastic electron-deuteron scattering is mainly sensitive to $G_{A}^{e}$.

The SAMPLE collaboration performed an experiment on a deuterium target as well as on a hydrogen target at $200 \mathrm{MeV}\left(Q^{2}=0.1(\mathrm{GeV} / c)^{2}\right)$. Combining the results from these two experiments allows separate determination of $G_{M}^{s}$ and $G_{A}^{e}$. The results from these measurements were published in Ref. [4]. Our data indicated that, while the overall contribution from strange quarks to the proton's magnetic form factor is small, the size of the electroweak radiative corrections (potentially including the anapole moment) to the axial form factor is significantly larger than anticipated from theory [5]. 
These results stimulated considerable interest among theorists. Many different processes and effects were studied for their potential contributions to the axial form factor, including the anapole moment [5-8], nuclear effects including two body currents [9], and the parity violating hadronic interaction [10,11]. Despite intensive efforts, the discrepancy between the theoretical prediction and the experimental value was not reconciled.

\section{SAMPLE III EXPERIMENT}

In order to experimentally confirm these results, we performed a third SAMPLE experiment, with a deuterium target at a lower beam energy of $125 \mathrm{MeV}$ $\left(Q^{2}=0.038(\mathrm{GeV} / c)^{2}\right)$. Just like the $200 \mathrm{MeV}$ experiment with a deuterium target, this experiment would mainly be sensitive to $G_{A}^{e}$. Since the PV asymmetry in the cross section is (to first order) proportional to $Q^{2}$, the expected asymmetry would be roughly 3 times smaller than that for $200 \mathrm{MeV}$. The cross section, however, is larger roughly by a factor of 2 , and since it was expected (and was later experimentally confirmed) that the background level for $125 \mathrm{MeV}$ would be the same as that for $200 \mathrm{MeV}$, we would expect roughly the same figure-of-merit for the $125 \mathrm{MeV}$ experiment as for the $200 \mathrm{MeV}$ experiment. Therefore, this experiment at $125 \mathrm{MeV}$ is an experiment that is sensitive to the same physics but with very different systematics.

The experiment was performed at the MIT Bates Linear Accelerator Center. The apparatus used for the SAMPLE III was identical to the one used for the previous SAMPLE experiments. A $125 \mathrm{MeV}$ longitudinally polarized electron beam was incident on a $40 \mathrm{~cm}$ long liquid deuterium target, and electrons that were scattered at backward angles were detected by a large solid angle air Čerenkov detector covering angles between $130^{\circ}$ and $170^{\circ}$. The beam was pulsed at $600 \mathrm{~Hz}$,and the average beam current was $40 \mu \mathrm{A}$. The polarized electron beam was generated at the Bates Polarized Electron Source by shining a circularly polarized laser beam onto a GaAs crystal. The helicity of the beam was randomly chosen for each pulse. The helicity of the beam (with respect to the electronics signal in the Polarized Source) was manually reversed every 2-3 days by inserting and removing a halfwave plate in the laser beam path to check and reduce possible systematic effects.

After a successful test run in April-May 2001 in which the expected background conditions were achieved, the production data were taken in November 2001-February 2002. About $96 \mathrm{C}$ of beam charge worth data was written on tape. In order to measure background components, $11 \%$ of the data were taken with the shutters (made of think aluminum sheet) in front of the PMT's closed to block the light coming into the PMT's.

\section{SAMPLE II ANALYSIS UPDATE}

In the mean time, the data analysis on the previous SAMPLE experiments (200 MeV run on hydrogen and deuterium targets) was continued. The background dilution factor was carefully reevaluated with the contribution from the coherent photoproduction of 
$\pi^{0}$ on the deuteron taken into account using new experimental results. Moreover, electromagnetic radiative effects were reevaluated with an improved detector acceptance model, and the theoretical value for the asymmetry with which the experimental value is to be compared was reevaluated with full nuclear calculation [12] and an improved detector acceptance model. The overall effect is such that the final physics asymmetry is increased (closer to the theoretical prediction) by $10 \%$ and the theoretical prediction for the asymmetry is decreased (closer to the experimental value) by $2 \%$. The implication of these updated results will be discussed at the end of the next section.

\section{SAMPLE III ANALYSIS PRESENT STATUS}

The data analysis was performed in a similar manner to the previous SAMPLE experiments. One of the differences from the analysis of the previous SAMPLE data is that the transmission (defined as the ratio of the beam intensity at the target to that at the end of the accelerator) was used as one of the parameters for the corrections procedure to correct for the intensity asymmetry due to the differential scraping at the energy defining slit.

In Fig. 1, the detector asymmetry is plotted as a function of time. The sign of the IN data is reversed. Correcting this raw asymmetry for the background dilution, the beam polarization, and electromagnetic radiative effects, we obtain the physics asymmetry. We then compare this physics asymmetry to the theoretical prediction, which was generated by averaging over the detector acceptance the results of the full nuclear calculation [12]. The comparison between the experimental asymmetry with the theoretical prediction is plotted in Fig. 2 for both SAMPLE II (updated results) and SAMPLE III. The data for both energies are consistent with the theoretical prediction with the value of $G_{A}^{e}$ taken from Ref. [5].

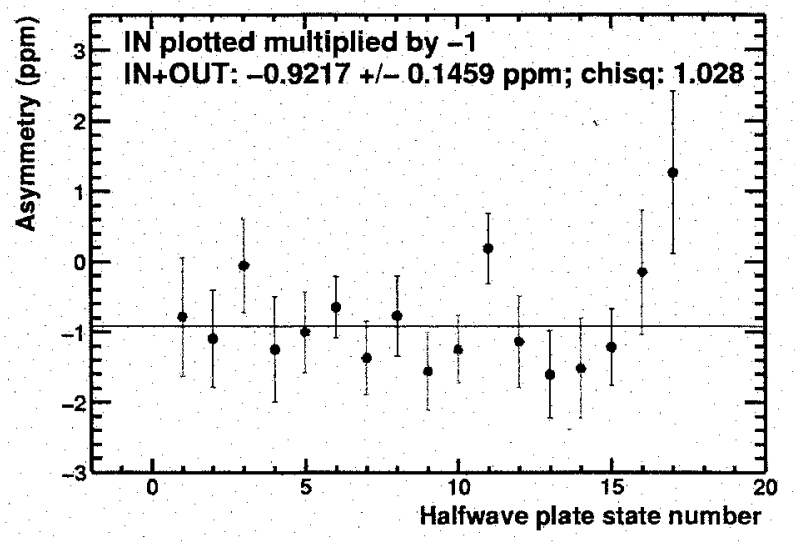

FIGURE 1. Results for the detector signal asymmetry for SAMPLE III. Each data point represents the average of the detector asymmetry between two halfwave plate state changes. The OUT data are plotted in red and the IN data are plotted in blue. The sign of the IN data is reversed. 


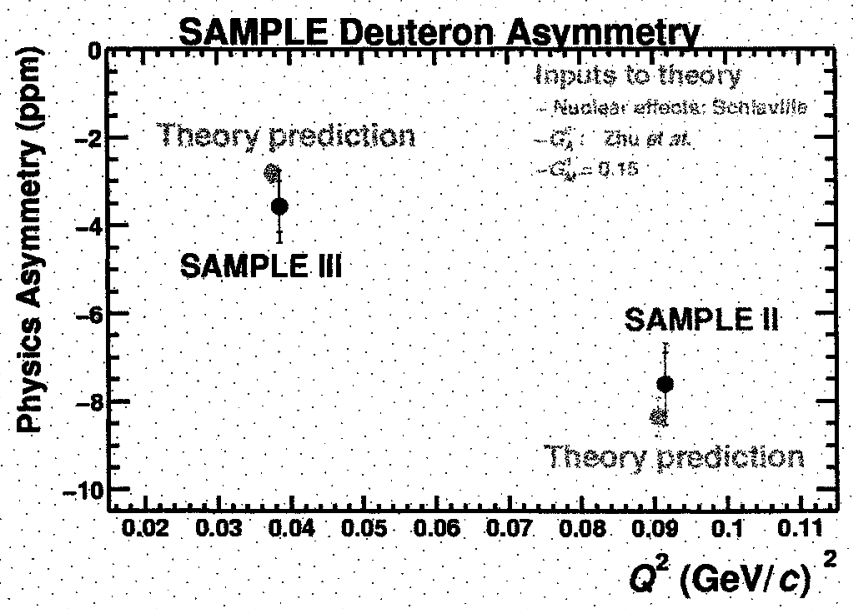

FIGURE 2. The physics asymmetry measured in SAMPLE II (updated results) and SAMPLE III are plotted as a function of $Q^{2}$. Also plotted are the theoretical prediction with the value of $G_{A}^{e}$ taken from [5], and $G_{M}^{s}=0.15$. The dependence of the theoretical values on the value of $G_{M}^{s}$ is small. The experimental values are consistent with the theoretical prediction.

\section{SUMMARY}

The updated results from the $200 \mathrm{MeV}$ SAMPLE deuterium run (SAMPLE II) and the results from the $125 \mathrm{MeV}$ SAMPLE deuterium run (SAMPLE III) both agree with the theoretical prediction on the electroweak radiative correction on the neutral weak axial form factor of the nucleon. In addition to these two experimental results, various theoretical efforts also support the theoretical predition on the electroweak radiative corrections on the axial form factor by Zhu et al. [5]. With these confirmation on the theoretical value of $G_{A}^{e}$, the theoretical value of $G_{A}^{e}$ can be used to extract $G_{M}^{s}$ from the data from the SAMPLE hydrogen run (SAMPLE ).

\section{REFERENCES}

1. R. D. McKeown, Phys. Lett. B 219, 140 (1989).

2. D. H. Beck, Phys. Rev. D 39, 3248 (1989).

3. D. B. Kaplan and A. Manohar, Nucl. Phys. B310, 527 (1988).

4. SAMPLE Collaboration, R. Hasty et al., Science, 290, 2021 (2000).

5. Shi-lin Zhu, S. J. Puglia, B. R. Holstein, and M. J. Ramsey-Musolf, Phys. Rev. D 62, 033008 (2000).

6. C. M. Maekawa, U. van Kolck, Phys. Lett. B 478, 73 (2000).

7. C. M. Maekawa, J. S. Veiga, U. van Kolck, Phys. Lett. B 488, 167 (2000).

8. D. O. Riska, Nucl. Phys. A678, 79 (2000).

9. L. Diaconescu, R. Schiavilla, U. van Kolck, Phys. Rev. C, 63, 044007 (2001).

10. R. Schiavilla, J. Carlson and M. Pais, Phys. Rev. C 67, 032501(R) (2003).

11. C.-P. Liu, G. Prézeau, and M. J. Ramsey-Musolf, Phys. Rev. C 67, 035501 (2003).

12. R. Schiavilla, Private communication (2003). 